\title{
Palliative Chemotherapy for Malignant Pheochromocytoma: Symptomatic Palliation of Two Cases
}

\author{
Masayasu Iwabuchi, Yutaka OKı and Hirotoshi NaKAmURA
}

\begin{abstract}
Malignant pheochromocytoma is a rare tumor with a poor prognosis because excess production of catecholamines leads to potentially lethal complications. Several chemotherapy regimens have been reported to be effective against this tumor, but a standard form of chemotherapy has not been established. We treated two patients with histologically confirmed pheochromocytoma after surgical removal of the primary lesion. Non-cardiogenic pulmonary edema was resolved and bone metastases were controlled by individualized chemotherapy that decreased the catecholamine levels, and the performance status was improved in both cases. Palliative chemotherapy should be designed to improve the quality of life of cancer patients.

(Internal Medicine 38: 433-435, 1999)
\end{abstract}

Key words: pulmonary edema, bone metastasis, cytosine arabinoside, individualized modification, quality of life

\section{Case Reports}

\section{Case 1}

A 35-year-old woman with malignant pheochromocytoma was admitted with dyspnea, hypertensive attacks, constipation, and a sensation of impending doom in 1993. Her blood pressure was $176 / 118 \mathrm{mmHg}$ and heart rate, $110 \mathrm{bpm}$. The oxygen saturation was $90 \%$. Abdominal X-ray films showed constipation and computed tomography showed pulmonary edema. Echocardiography showed a normal ejection fraction.

In 1988, she had undergone surgical treatment of a pheochromocytoma in the bladder wall and angiography had shown multiple lymph node metastases in the pelvis, indicating the malignancy of her disease.

Investigations revealed elevated catecholamine levels and disease progression. However, no mass lesions were found except for equivocal swelling of lymph nodes in the pelvis. The plasma norepinephrine level was $41 \mathrm{ng} / \mathrm{ml}$ (normal: 0.05-0.40) and 24hour urinary norepinephrine was $17,100 \mu \mathrm{g} / \mathrm{day}$ (normal: 29-120). There was no uptake of 131-metaiodobenzylguanidine (MIBG) on scanning, suggesting that MIBG therapy was not indicated. Adrenergic blockade did not relieve her symptoms, so cytoreductive therapy was tried. The chemotherapeutic regimen, consisting of $750 \mathrm{mg} / \mathrm{m}^{2}$ of cyclophosphamide on day $1,1.4$ $\mathrm{mg} / \mathrm{m}^{2}$ of vincristine on day 1 , and $600 \mathrm{mg} / \mathrm{m}^{2}$ of dacarbazine on days 1 and 2 (CVD), has been reported to be effective against malignant pheochromocytoma $(1,2)$. Due to her poor general condition, we modified the CVD regimen to VdsD; cyclophosphamide was not administered, $6 \mathrm{mg} / \mathrm{m}^{2}$ of vindesine was given in place of vincristine on day 1 , and dacarbazine was given according to the original schedule. She received chemotherapy at approximately 10-day intervals with careful observation of toxicity. Her pulmonary edema was resolved (Fig. 1A, B) and bowel function improved, with a decrease of 24-hour urinary catecholamines (Fig. 1C). The sensation of impending doom also diminished. According to the Eastern Cooperative Oncology Group, her performance status was improved from grade 3 to 1 . She was discharged and continued to receive chemotherapy for 15 months as an outpatient. In 1995, seven years after the diagnosis of malignant pheochromocytoma, she died of cerebral hemorrhage due to disease progression.

\section{Case 2}

A 67-year-old man underwent resection of a pheochromocytoma of the right adrenal gland in 1986. Malignancy was diagnosed after multiple metastases to the paraaortic nodes were detected in 1992. Three courses of CVD did not produce improvement of catecholamine levels. He was followed as an outpatient, and lumbar vertebral metastases were treated with 40 Gy of radiation in 1995. In 1996, he was admitted because of persistent back pain and aspiration pneumonia due to recurrent laryngeal nerve paralysis caused by disease progression. A Tc-99m bone scan showed abnormal hot spots in L5 and the right fifth rib ( Fig. 2A). Metastases were also observed in the

From the Second Division, Department of Medicine, Hamamatsu University School of Medicine, Hamamatsu

Received for publication September 29, 1998; Accepted for publication January 8, 1999

Reprint requests should be addressed to Dr. Masayasu Iwabuchi, the Second Division, Department of Medicine, Hamamatsu University School of Medicine, Hamamatsu 3600, Handa-cho, Hamamatsu 431-3124 
B

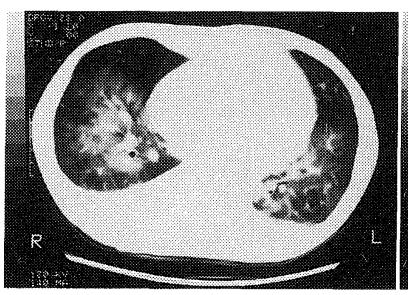

C

$(\mu \mathrm{g} /$ day $)$

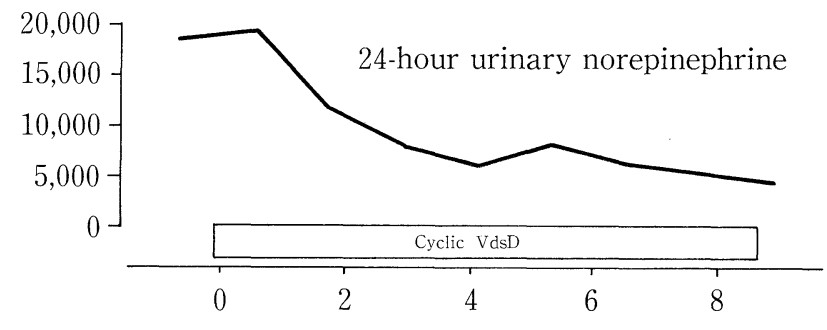

Weeks after starting chemotherapy

Figure 1. Improvement of pulmonary edema and 24-hour urinary norepinephrine excretion. Chemotherapy was performed with $6 \mathrm{mg} / \mathrm{m}^{2}$ of vindesine on day 1 , and $600 \mathrm{mg} / \mathrm{m}^{2}$ of dacarbazine on days 1 and 2 (VdsD) at approximately 10-day intervals. A) Before chemotherapy, pulmonary edema is evident on computed tomography. B) Three weeks after starting chemotherapy, pulmonary edema has resolved. C) Twenty-four-hour urinary norepinephrine excretion was decreased by treatment.

lung field and the paraaortic region. Pain control with morphine was attempted, but failed. Subcutaneous administration of $20 \mathrm{mg}$ of cytosine arabinoside was performed twice a week ( $1 \mathrm{mg} / \mathrm{kg} /$ week) under Adrenergic blockade. Four weeks later, his bone pain was resolved, and a repeat Tc-99m bone scan showed improvement (Fig. 2B). The 24-hour urinary norepinephrine excretion was decreased by $90 \%$ ( from $22,600 \mu \mathrm{g}$ ) day to 2,140 $\mu \mathrm{g} /$ day; Fig. 2C). Low-dose cytosine arabinoside was continued for 6 months and his performance status improved from grade 3 to 2 . In December 1996, he died of respiratory failure at 4 years after the diagnosis of malignant pheochromocytoma.

\section{Discussion}

In patients with pheochromocytoma, objective remission means the regression of measurable tumor lesions to less than $50 \%$ and a decrease in catecholamine concentration and disease-related symptoms (3). It has been reported that reducing the catecholamine level achieves palliation of malignant pheochromocytoma (4). The present two cases did not show significant regression of measurable tumors but did obtain symptomatic improvement. In case 1 , modified CVD therapy con-
A
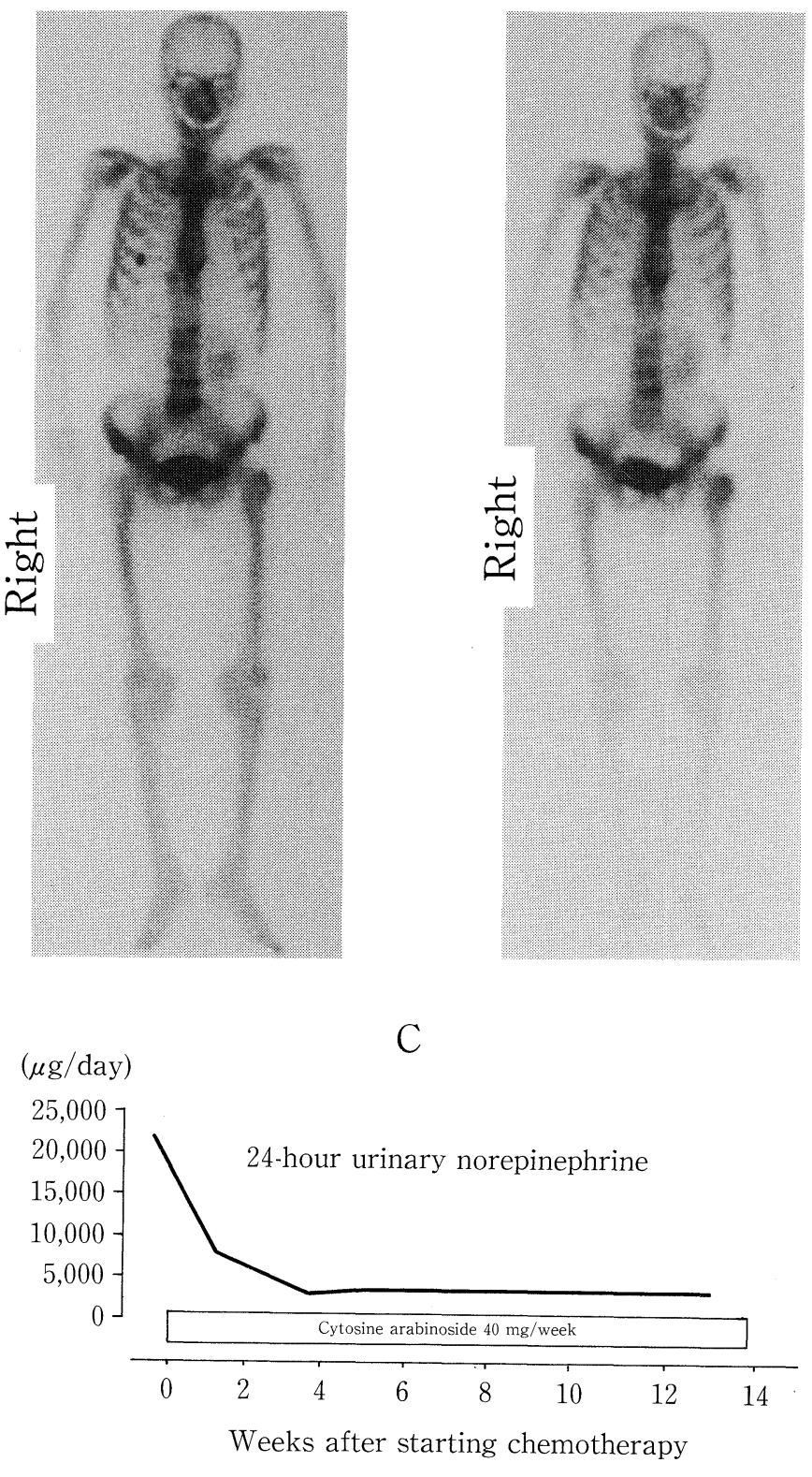

Figure 2. Bone scan findings and 24-hour urinary norepinephrine excretion. A, B) Tc-99m bone scans show a decrease of hot spots after chemotherapy. A) Before chemotherapy. B) After 2 months of chemotherapy. C) Twenty-four-hour urinary norepinephrine excretion was markedly decreased by treatment.

trolled the pulmonary edema and other symptoms. An elevated level of catecholamines can cause pulmonary edema by increasing pulmonary capillary permeability and peripheral vasocontriction, leading to a shift of extracellular fluid (5). In addition, catecholamines reduce bowel motility, leading to paralytic ileus. The chemotherapy regimen we used was modified to avoid hydration-induced heart failure and to reduce the risk of vinca alkaloid neuropathy (6); the vindesine dose was de- 
termined from the sensitivity testing of cultured human neuroblastoma cells (7), and the treatment schedule was derived from a study of chemotherapy-induced bone marrow changes in a neuroblastoma patient (8). There was a clear decrease of the norepinephrine level in case 1 , associated with symptomatic improvement. Hypertensive episodes due to pheochromocytoma are sometimes accompanied by a sense of impending doom, and control of such attacks gives the patient a sense of well-being.

Starling and coworkers reported that neuroblastomas resistant to cyclophosphamide and vincristine did not respond to cytosine arabinoside (9). Our case 2 did not want to undergo intensive chemotherapy, although morphine and radiotherapy had not controlled his lumbar pain. Fortunately, low-dose cytosine arabinoside achieved palliation without drug toxicity, and he spent 6 more pain-free months with his wife after his performance status was improved from grade 3 to 2. Singleagent treatment of malignant pheochromocytoma has not produced survival longer than 4 years (10). The benefits of lowdose cytosine arabinoside for hematologic disorders have been reported $(11,12)$, this is the first report of malignant pheochromocytoma treated with such therapy. Though it was reported that more than $85 \%$ of the patients with malignant pheochromocytoma die with a median survival of 16 months from diagnosis of metastases (13), our patients obtained longer survival than has been reported. Moreover, they obtained improvement in symptoms and performance status according to the Eastern Cooperative Oncology Group, suggesting that individualized chemotherapy is useful for palliation of malignant pheochromocytoma, and that successful control of potentially lethal complications may improve the prognosis of the tumor.

Acknowledgements: We would thank Dr. Toshifumi Katsuki (Yaizu Municipal Hospital), and Dr. Toru Watanabe (National Cancer Center) for their invaluable advice and the E10 staff for special nursing.

\section{References}

1) Keiser HR, Goldstein DS, Wade JL, Douglas FL, Averbuch SD. Treatment of malignant pheochromocytoma with combination chemotherapy. Hypertension 7: I18-I24, 1985.

2) Averbuch SD, Steakley CS, Young RC, et al. Malignant pheochromocytoma: Effective treatment with a combination of cyclophosphamide, vincristine, and dacarbazine. Ann Intern Med 109: 267-273, 1988.

3) WHO handbook for reporting results of cancer treatment. Geneva. World Health Organization, 1979.

4) Senan S, Reed N, Connell J. Palliation of malignant phaechromocytoma with combination chemotherapy. Eur J Cancer 28A: 1007-1008, 1992.

5) Sjoerdsma A, Engelman K, Waldmann TA, et al. Pheochromocytoma: current concepts of diagnosis and treatment. Combined clinical staff conference at the National Institute of Health. Ann Intern Med 65: 1302 1326, 1966.

6) Matsushita A, Takesue H, Ikeda M, et al. General pharmacology of vindesine sulfate. (3) Changes in motor behaviors and muscle spindle activities of the cat following chronic administration of vindesine and vincristine. Ouyou Yakuri 25: 655-662, 1983 (in Japanese).

7) Hill BT, Whelan RD. Assessment of the sensitivities of cultured human neuroblastoma cells to anti-tumor drugs. Pediatr Res 15: 1117-1122, 1981.

8) Shackney SE, Bunn PA, Ford SS, Erickson B, Ross WE, Levine AS. A study of drug-induced kinetic perturbations in the marrow of a patient with neuroblastoma. Cancer 45: 882-892, 1980.

9) Starling KA, Sutow WW, Donaldson MH, Land VJ, Lane DM. Drug trials in neuroblastoma: cyclophosphamide (NSC-26271) alone; vincristine (NSC-67574) plus cyclophosphamide; 6-mercaptopurine (NSC-755) plus 6-methylmercaptopurine riboside (NSC-40774); and cytosine arabinoside (NSC-63878) alone. Cancer Chemother Rep 58: 683-688, 1974.

10) Siddiqui MZ, Von Eyben FE, Spanos G. High-voltage irradiation and combination chemotherapy for malignant pheochromocytoma. Cancer $\mathbf{6 2}$ : 686-690, 1988.

11) Baccarani M, Tura S. Differentiation of myeloid leukaemic cells: new possibilities for therapy. Br J Haematol 42: 485-487, 1979.

12) Housset M, Daniel MT, Degos L. Small doses of ARA-C in the treatment of acute myeloid leukemia: differentiation of myeloid leukaemia cells? Br J Haematol 51: 125-129, 1982.

13) Schlumberger M, Gicquel C, Lumbroso J, et al. Malignant pheochromocytoma: clinical, biological, histologic and therapeutic data in a series of 20 patients with distant metastases. J Endocrinol Invest 15: 631-642, 1992. 